\title{
In-Line Half-Kneeling as a Motor Control Test of Core Stability: Known-Groups Validity and Reliability
}

\author{
David M. Werner and Joaquin A. Barrios
}

\begin{abstract}
Context: Core stability is considered critical for the successful execution of rehabilitative and athletic tasks. Although no consensus definition exists, different components related to core stability have been identified. An important component is the domain of motor control. There are few clinical tests assessing the motor control component of core stability (MCCS). Objective: To evaluate the interrater reliability and known-groups validity of a novel test of MCCS, the in-line half-kneeling test. The test is aimed at assessing MCCS by challenging the ability to maintain a static position with minimized contributions from the distal extremities over a minimized base of support. Design: Cross-sectional group comparison study. Setting: Laboratory. Patients or Other Participants: A total of 75 participants (25 individuals with a history of anterior cruciate ligament reconstruction, 25 uninjured Division 1 collegiate athletes, and 25 uninjured controls) were recruited from a university community. Intervention: Participants were video recorded while performing the in-line half-kneeling test for 120 seconds bilaterally. Three observers independently viewed each video to determine if individuals broke form during each test using 2 dichotomous criteria. Main Outcome Measures: Cohen's kappa was used to assess interrater reliability, and chi-square tests of independence were used to compare break rates between groups. Results: Good-to-excellent interrater reliability (.732-.973) was seen between the 3 observers. Chi-square tests of independence revealed different break rates between all 3 groups. Compared to break rate for the reference control group (11/25-44\%), those with a history of anterior cruciate ligament reconstruction broke at a higher rate $(18 / 25-72 \%)$, whereas the uninjured collegiate athletes broke at a lower rate (4/25-16\%). Conclusions: The in-line halfkneeling test is a reliable test between raters that can differentiate between groups expected to differ in MCCS.
\end{abstract}

Keywords: anterior cruciate ligament, clinical evaluation, trunk, proprioception, neuromuscular

Core stability is considered fundamental for optimized wholebody movement. Functionally, core stability is partly reflected in the ability of the trunk to maintain or return the body to equilibrium when challenged by both expected and unexpected internal and external perturbations. ${ }^{1,2}$ While no consensus definition exists, core stability has been described as having distinct testable components, including strength, endurance, flexibility, function, and motor control. Tests for functional stability under perturbation can directly challenge the motor control component of core stability (MCCS) ${ }^{3,4}$ Currently, few assessments for MCCS have been put forth in the literature.

In-line half-kneeling (ILHK) is proposed as an MCCS test. This bilateral test involves maintaining static half-kneel positions without breaking form. The purpose of the test is to evaluate positional stability in response to predominantly internal perturbations while constraining the influences of mediolateral base of support, visual feedback, and upper-extremity strategies. The aims of this study were twofold: first, to assess the interrater reliability of video assessment, and second, to use a known-groups validation approach to compare break frequencies between 3 populations that might be expected to differ in MCCS.

\section{Methods}

\section{Participants}

Participants were recruited from a university setting and provided informed consent as approved by the University of Dayton's Institutional Review Board. All participants were currently healthy

The authors are with the Department of Physical Therapy, University of Dayton, Dayton, OH, USA. Werner (wernerd1@udayton.edu) is corresponding author. and between 18 and 45 years old. Three groups of equal numbers $(\mathrm{N}=75)$ were recruited: healthy normal individuals, collegiate athletes, and individuals with a history of ACLR. The athlete group consisted of participants currently rostered on a Division I sports team. The ACLR group had all undergone ACLR greater than 12 months prior, completed formal rehabilitation, and received physician clearance for unrestricted physical activity. Participants were excluded if they presented with injuries or disease to the lowerextremity and/or spine within the previous 12 months.

\section{Procedures}

The testing area included a 2-m piece of tape (Kendall Waterproof Tape; Tyco Healthcare Group, Mansfield, MA), a video recording device (720P FaceTime HD camera, MacBook Pro; Apple, Cupertino, CA) positioned on a $30-\mathrm{cm}$ tall box located $1.5 \mathrm{~m}$ in front of the end of the tape, and a small piece of tape placed on a wall $9 \mathrm{~m}$ away and $1.35 \mathrm{~m}$ high as a visual target.

Participants were shown a video and then viewed a demonstration of the ILHK position (Figure 1). Participants were then given standardized instructions.

1. Front foot centered on tape with toes at the end of the tape

2. $90^{\circ}$ angles of front hip and knee

3. Back knee (on a twice-folded hand towel) at $90^{\circ}$

4. Dorsum (top) of back foot on line

5. Gaze fixed on piece of tape ahead

6. Balanced position with arms crossed and close to body

7. If position is lost, reassume test position and continue until notified by examiner 
Based on pilot data, participants were video recorded for 120 consecutive seconds bilaterally with 120 -second rest between sides. Test order of limbs was counterbalanced by dominance, with the limb-bearing weight through the knee considered the test limb.

Three trained, independent observers separately scored each video using break/no break criteria. Observers were trained on 4 pilot videos. When scoring, observers were freely allowed to zoom, slow, pause, and replay the videos. A form break was logged if one or both criteria were met: (1) The front foot fully displaced from the tape, showing complete visible separation between the entire front foot and the tape or (2) either or both hands left the torso. Other movements were not considered form breaks. If participants successfully completed 120 seconds without breaking, the unilateral effort was classified as a "no break" and the other side was tested identically. Participants were placed in the "break" classification if they had a form break at any time during the cumulative 240 seconds of bilateral testing.

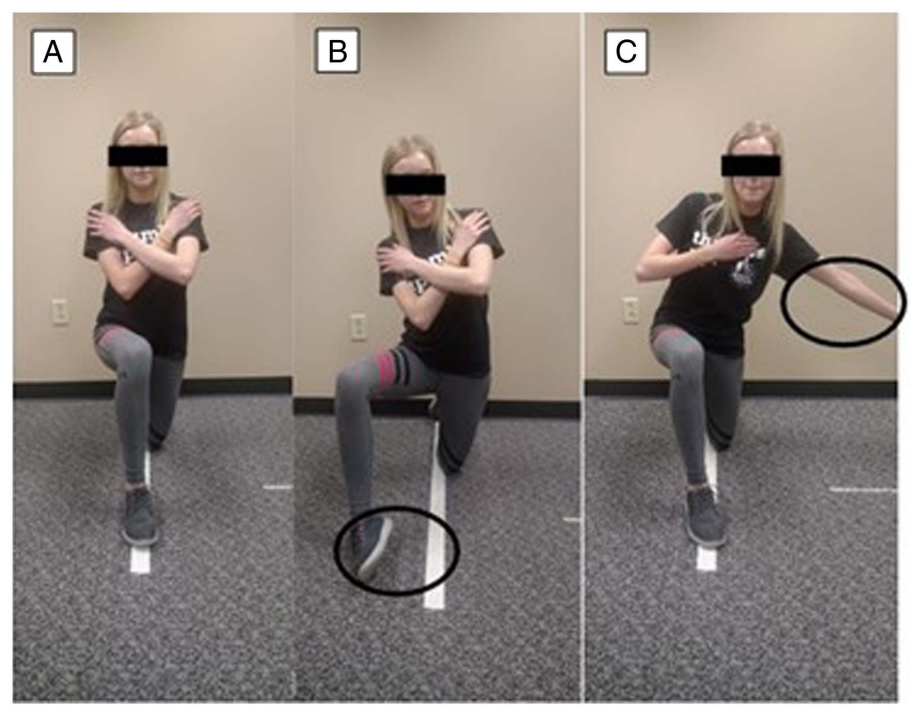

Figure 1 - Performance of the in-line half-kneeling test. (A) Frontal view of correct form. (B) Form break: separation between foot and tape. (C) Form break: one hand removed from torso.

\section{Statistical Analyses}

Statistical analyses were performed using SPSS (version 20.0; SPSS Inc, Chicago, IL). Group descriptive data were expressed as either frequency counts or means and standard deviations. Homogeneity between groups in age, height, weight, and body mass index were assessed using single-factor analysis of variance. Chi-square was used to assess sex distributions. Interrater reliability was assessed with Cohen's kappa, using data from all participants. The break rate analyses were conducted using the data from the first observer. To compare break frequencies between groups, a $3 \times 2$ chi-square test of independence was conducted. Post hoc testing of a significant chi-square was performed using assessment of standardized residuals. If standardized residuals were \pm 1.96 from the expected frequency (determined from the break frequency of the control participants), it represented the major cells contributing to a significant chi-square. ${ }^{5}$ An alpha level of .05 was used.

\section{Results}

Groups did not differ by age, height, weight, body mass index, or sex distribution $(P>.05$; Table 1$)$.

Interrater reliability was good to excellent (.732-.973) among the 3 observers (Table 2). The chi-square test of independence $(P<.001)$ suggested break rate differed by group. For the post hoc assessment, the standardized residual of the break frequencies for the athletes was -2.1 , whereas the ACLR group's standardized residual was +2.1 . These findings indicated that the break rates for the athlete (16\%) and ACLR (72\%) groups were different from the break rate of the healthy normal participants (44\%) (Table 3).

\section{Discussion}

The results of this study support the utility of ILHK as a test of MCCS. Good-to-excellent interrater reliability was seen across 3 observers assessing video playback. Furthermore, ILHK differentiated 3 populations by likelihood of positional breaking.

The interrater reliability of tests aimed at assessing MCCS appears variable. Weir et $\mathrm{al}^{6}$ found poor interrater reliability across 6 tests, including unilateral squatting, lateral step-downs, and bridging, regardless of utilizing a 4-point or 2-point scoring criterion (intraclass correlation coefficient $[\mathrm{ICC}]_{2,1}=.09-.51$ ).

Table 1 Descriptive Characteristics for Healthy Controls, NCAA Athletes, and ACLR Subjects (Frequency Counts or Mean [SD])

\begin{tabular}{lcccc}
\hline & Healthy & Athletes & ACLR & P value \\
\hline Sex (female:male) & $21: 4$ & $17: 8$ & $20: 5$ & .37 \\
Age, y & $21.2(1.8)$ & $20.5(1.4)$ & $20.4(1.8)$ & .68 \\
Height, m & $1.7(0.1)$ & $1.7(0.1)$ & $1.7(0.1)$ & .77 \\
Weight, kg & $63.7(12.4)$ & $69.9(14.4)$ & $68.1(11.4)$ & .59 \\
BMI, kg/m & $22.8(3.1)$ & $22.9(3.0)$ & $23.2(2.2)$ & .90 \\
Time since surgery, mo & & & $44.5(27.5)$ & \\
Other ligament injury & & 4 MCL:2 PCL & \\
Meniscus tear & & & 19 & \\
Graft type (HS:PT:Allo) & & & $9: 12: 4$ & \\
\hline
\end{tabular}

Abbreviations: ACLR, Anterior Cruciate Ligament Reconstruction; Allo, allograft; BMI, body mass index; HS, hamstrings; MCL, medial collateral ligament; NCAA, National Collegiate Athletic Association; PCL, posterior cruciate ligament; PT, patellar tendon. 
Table 2 Point-to-Point Percent Agreement and Interrater Reliability $(\mathrm{N}=75)$

\begin{tabular}{lccc}
\hline & $\begin{array}{c}\text { No break } \\
\text { \% agreement }\end{array}$ & $\begin{array}{c}\text { Break } \\
\text { \% agreement }\end{array}$ & $\begin{array}{c}\text { Cohen's } \\
\text { kappa }\end{array}$ \\
\hline O1 versus O2 & 100 & 97 & .973 \\
O1 versus O3 & 92 & 84 & .763 \\
O2 versus O3 & 90 & 83 & .736 \\
\hline
\end{tabular}

Abbreviation: $\mathrm{O}$, observer.

Table 3 Between-Group Comparisons

\begin{tabular}{lcc}
\hline & No break & Break \\
\hline Healthy & 14 & \\
Count & 14 & 11 \\
Expected count & 0 & 11 \\
Standardized residual & $56 \%$ & 0 \\
Rate & & $44 \%$ \\
Athlete & 21 & \\
Count & 14 & 4 \\
Expected count & +1.9 & 11 \\
Standardized residual & $84 \%$ & $-2.1 *$ \\
Rate & & $16 \%$ \\
ACLR & 7 & 18 \\
Count & 14 & 11 \\
Expected count & -1.9 & $+2.1 *$ \\
Standardized residual & $28 \%$ & $72 \%$ \\
Rate & & \\
\hline
\end{tabular}

*Statistically significant.

By contrast, Monnier et $\mathrm{al}^{7}$ found good-to-excellent interrater reliability (ICC $=.56-.95)$ for 6 active movement control tests using binary scoring. Noehren et $\mathrm{al}^{8}$ found excellent interrater reliability $\left(\mathrm{ICC}_{3, k}=.93\right)$ when assessing the frequency of 6 welldefined failures during the trunk stability test (TST). Combining the current results and previous research, it appears that attaining goodto-excellent interrater reliability for a measure of MCCS is often contingent on 2 factors: well-defined scoring criteria and a scoring system with fewer outcome classifications.

The data supported the hypothesis that a group with a history of ACLR would break at a higher frequency than a control group. These results are similar to Noehren et al, ${ }^{8}$ who compared individuals with a history of ACLR to healthy individuals and observed impaired trunk control when performing the TST. Participants sat in a standardized position on an exercise ball while holding their contralateral limb off the ground with eyes closed for 30 seconds. ${ }^{8}$ Those with ACLR demonstrated more failures. Both the TST and the ILHK test purport to increase proximal demand while controlling one's body over a narrow base of support. ILHK may be advantageous to the TST in that testing does not involve equipment nor require nearby objects to prevent falls, and it has only 2 criteria to evaluate.

At least 2 factors related to proximal neuromuscular control could help explain the observed higher break rate after ACLR. First, potential deficits in trunk proprioception in the ACLR group may have affected their ability to detect losses in positional stability. The narrow base of support likely accentuates any existing deficits in trunk proprioception. A second potential factor is that individuals with ACLR possibly have reduced proximal neuromuscular capacity to execute effective recovery strategies, once losses in positional stability are perceived. In a prospective injury trial, deficits in trunk neuromuscular control were found to predict lower-extremity injury risk, including primary ACL rupture. ${ }^{2}$ In that study, the authors suggest that the ability to correct body sway in the coronal plane is relevant in core motor control.

The lower break rate of the athletes also supported the initial hypotheses. Previous studies have shown that athletes perform better on tests assessing MCCS compared with nonathletes. Highlevel athletes tested on a stabilometer, as well as National Collegiate Athletic Association (NCAA) Division 1 athletes on a Biodex balance system (Biodex Medical Systems Inc, Shirley, NY), have demonstrated better postural sway performance on unstable surfaces compared with untrained and nonathlete controls. ${ }^{9,10}$ These findings relate to the current study in that ILHK also requires maintaining positional stability over a compromised base of support. The lower break rate in athletes may relate to the demands for stability during dynamic and highly loaded tasks that they often routinely experience.

Clinical translation of these ILHK methods is straightforward, with one minor recommended modification. Specifically, any single break on either limb terminates the test, and the "break" classification is assigned rather than having the subject continue the test. We recommend this modification based on the proof-of-concept nature of this study. Furthermore, live visual scoring rather than using video playback may have a greater clinical ease of utility.

The overall aim of this study was to propose ILHK as a test of MCCS. The results suggest that test scoring has good-to-excellent interrater reliability. Furthermore, the test was able to distinguish groups with varying a priori expectations in MCCS. The ILHK test appears to have clinical utility as a test for MCCS.

\section{References}

1. Pope MH, Panjabi M. Biomechanical definitions of spinal instability. Spine. 1985;10(3):255-256. PubMed ID: 3992345 doi:10.1097/ 00007632-198504000-00013

2. Zazulak BT, Hewett TE, Reeves NP, Goldberg B, Cholewicki J. Deficits in neuromuscular control of the trunk predict knee injury risk: a prospective biomechanical-epidemiologic study. Am J Sports Med. 2007;35(7):1123-1130. PubMed ID: 17468378 doi:10.1177/ 0363546507301585

3. Waldhelm A, Li L. Endurance tests are the most reliable core stability related measurements. J Sport Health Sci. 2012;1(2):121-128. doi: 10.1016/j.jshs.2012.07.007

4. Parkhurst TM, Burnett CN. Injury and proprioception in the lower back. J Orthop Sports Phys Ther. 1994;19(5):282-295. PubMed ID: 8199622 doi:10.2519/jospt.1994.19.5.282

5. Sheskin DJ. Handbook of Parametric and Nonparametric Statistical Procedures. New York, NY: CRC Press; 2003.

6. Weir A, Darby J, Inklaar H, Koes B, Bakker E, Tol JL. Core stability: inter- and intraobserver reliability of 6 clinical tests. Clin J Sport Med. 2010;20(1):34-38. PubMed ID: 20051732 doi:10.1097/JSM. 0b013e3181cae924

7. Monnier A, Heuer J, Norman K, Äng BO. Inter- and intra-observer reliability of clinical movement-control tests for marines. BMC Musculoskelet Disord. 2012;13(1):263. doi:10.1186/1471-247413-263

8. Noehren B, Abraham A, Curry M, Johnson D, Ireland ML. Evaluation of proximal joint kinematics and muscle strength following ACL 
reconstruction surgery in female athletes. J Orthop Res. 2014;32(10): 1305-1310. PubMed ID: 25044305 doi:10.1002/jor.22678

9. Davlin CD. Dynamic balance in high level athletes. Percept Mot Skills. 2004;98(3, pt 2):1171-1176. PubMed ID: 15291203 doi:10. 2466/pms.98.3c.1171-1176
10. Palmer TB, Hawkey MJ, Thiele RM, et al. The influence of athletic status on maximal and rapid isometric torque characteristics and postural balance performance in division I female soccer athletes and non-athlete controls. Clin Physiol Funct Imaging. 2015;35(4): 314-322. PubMed ID: 24890050 doi:10.1111/cpf.12167 